\title{
openheart Reintroduction of elective cardiac interventions in the era of COVID-19: the Barts experience
}

\author{
Stephen Hamshere (D) , ${ }^{1}$ Katrina Comer, ${ }^{1}$ Fizzah Choudhry, ${ }^{1,2}$ Krishna Rathod, ${ }^{1,2}$ \\ Gordon Mills, ${ }^{1}$ Gordon Ferguson, ${ }^{1}$ Jonathan Lambourne, ${ }^{3}$ Majid Akhtar, ${ }^{1}$ \\ Andrew Wragg, ${ }^{1}$ Mick Ozkor, ${ }^{1}$ Oliver Guttmann, ${ }^{1,2}$ Michael Mullen (i) ,1,2 \\ Andreas Baumbach, ${ }^{1,2}$ Elliot Smith, ${ }^{1,2}$ Anthony Mathur, ${ }^{1,2}$ Dan Jones ${ }^{1,2}$
}

To cite: Hamshere S, Comer K, Choudhry F, et al. Reintroduction of elective cardiac interventions in the era of COVID-19: the Barts experience. Open Heart 2021;8:e001446. doi:10.1136/ openhrt-2020-001446

Received 13 September 2020 Revised 29 0ctober 2020 Accepted 7 December 2020

A) Check for updates

(C) Author(s) (or their employer(s)) 2021. Re-use permitted under CC BY-NC. No commercial re-use. See rights and permissions. Published by BMJ.

${ }^{1}$ Barts Heart Centre, Barts Health NHS Trust, London, UK ${ }^{2}$ Centre for Cardiovascular Medicine and Devices, William Harvey Research Institute, NIHR Cardiovascular Biomedical Research Unit at Barts, Queen Mary University of London, London, UK

${ }^{3}$ Department of Infectious Diseases, Barts Health NHS Trust, London, UK

Correspondence to Dr Stephen Hamshere; stephenhamshere@doctors. org.uk

\section{ABSTRACT}

Background The response to COVID-19 has required cancellation of all but the most urgent procedures; there is therefore a need for the reintroduction of a safe elective pathway.

Methods This was a study of a pilot pathway performed at Barts Heart Centre for the admission of patients requiring elective coronary and structural procedures during the COVID-19 pandemic (April-June 2020). All patients on coronary and structural waiting lists were screened for procedural indications, urgency and adverse features for COVID-19 prognosis and discussed at dedicated multidisciplinary teams. Dedicated admission pathways involving preadmission isolation, additional consent, COVID-19 PCR testing and dedicated clean areas were used.

Results 143 patients (101 coronary and 42 structural) underwent procedures (coronary angiography, percutaneous coronary intervention, transcatheter aortic valve intervention and MitralClip) during the study period. The average age was $68.2 ; 74 \%$ were male; and over $93 \%$ had one or more moderate COVID-19 risk factors. All patients were COVID-19 PCR negative on admission with (8.1\%) COVID-19 antibody positive (swab negative). All procedures were performed successfully with low rates of procedural complications (9.8\%). At 2-week followup, no patients had symptoms or confirmed COVID-19 infection with significant improvements in quality if life and symptoms.

Conclusion We demonstrated that patients undergoing coronary and structural procedures can be safely admitted during the COVID-19 pandemic, with no patients contracting COVID-19 during their admission. Reassuringly, patients reflective of typical practice, that is, those at moderate or higher risk, were treated successfully. This pilot provides important information applicable to other settings, specialties and areas to reintroduce services safely.

\section{INTRODUCTION}

The outbreak of the COVID-19 has affected nearly fivemillion people worldwide, of which 319960 have died as of 18 May $2020{ }^{1}$ During the COVID-19 pandemic, pathways

\section{Key questions}

What is already known about this subject?

- COVID-19 has swept the world, resulting in a large number of deaths. Patients who have cardiovascular disease are a greater risk of mortality if contracting COVID-19. During the COVID-19 outbreak, there was a suspension of all elective activity both in cardiology and other specialties. There was a need for the reintroduction of a safe pathway for elective patients who required both coronary and structural cardiac procedures.

What does this study add?

- This study demonstrated that the reintroduction of a specifically designed pathway that protects patients and reduces their risk of exposure to COVID-19 can be safely implemented, with no patients acquiring COVID-19 during their treatment.

How might this impact on clinical practice?

- This study has important implications for the reintroduction of elective services during and after the COVID-19 pandemic, both in patients with cardiovascular and other diseases requiring treatment. This is the first study to show that elective procedures can be safely provided with appropriate precautions.

for treating cardiovascular disease have been disrupted, with elective activity discontinued to protect and reduce the risk to patients of contracting COVID-19. Additionally, there has been an expansion of high-dependency beds within hospitals, with the creation of 'COVID-19' isolation wards and redistribution of resources to treat the influx of patients with COVID-19. ${ }^{2}$ Untreated patients with established cardiovascular disease are in highrisk categories (eg, severe aortic stenosis) and therefore are at risk of deterioration, resulting in emergency admission or sudden cardiac death. Emergency admission is likely to place the patient at increased risk of contracting 
COVID-19. Delayed treatment will have consequences for recovery and long-term outcome in these patients, but many patients are appropriately deeply concerned about contracting COVID-19 in the hospital. These patients are also typically shielding, which may lead to delays in them seeking medical treatment with a reluctance to agree to admission even in the face of advanced symptoms and significant risk of clinical deterioration. There is therefore an imperative to maintain normal interventional treatments particularly for patients with prognostic disease. With the relaxation in social isolation, a need for the reintroduction of pathways for the urgent treatment of appropriate high-risk patients on elective waiting lists is required and with it the essential need to minimise the risk of patients contracting COVID-19.

The aim of this study was to describe a pilot pathway to facilitate the admission of urgent elective cardiology patients for the treatment of cardiovascular disease while minimising the risks of exposing them to SARCoV-2 infection during the COVID-19 pandemic. The

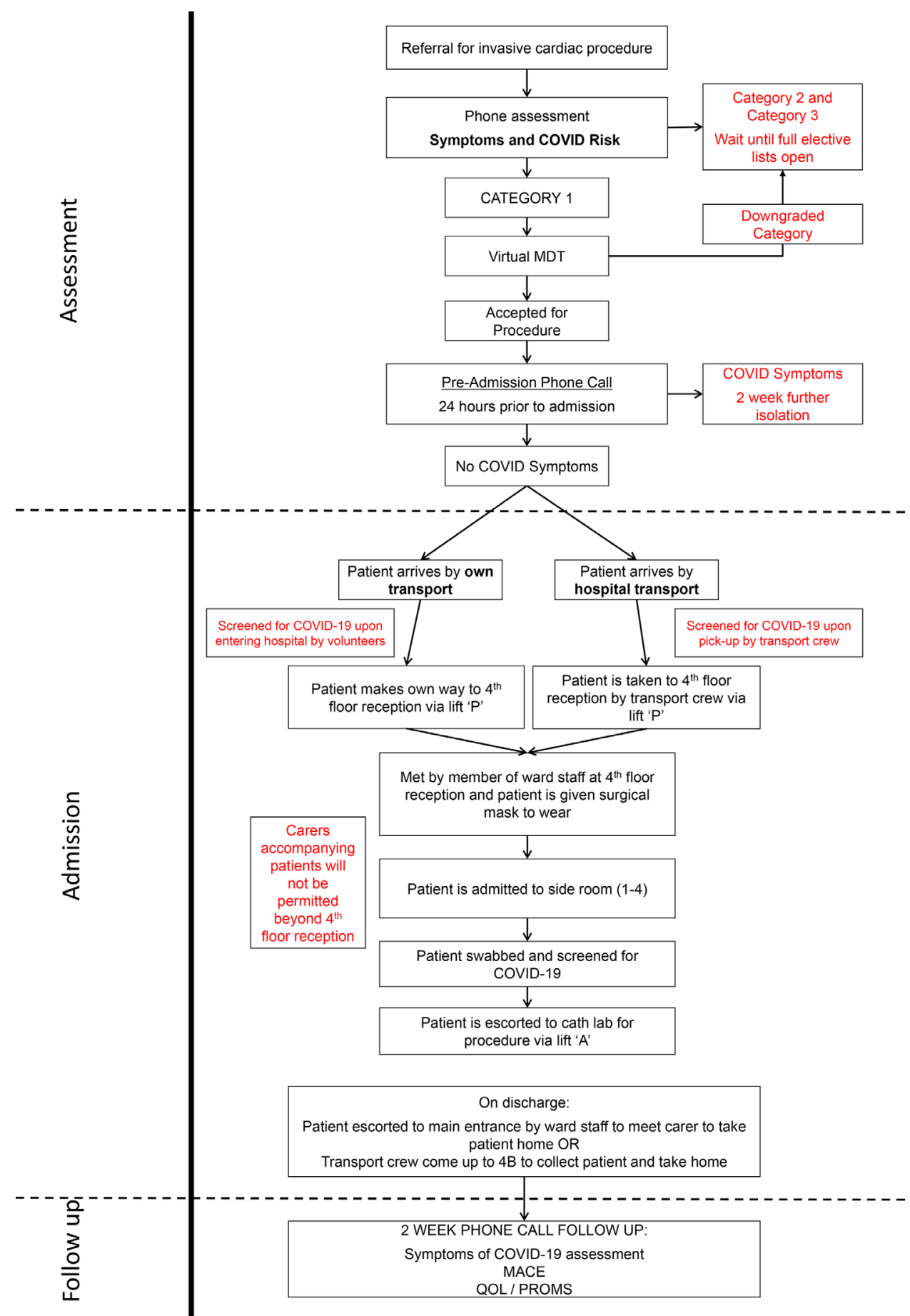

Figure 1 Patient flow diagram. MDT, multidisciplinary team; PROMS; Patient Reported Outcome Measures; QOL, quality of life. 
Table 1 Waiting list referrals

$\begin{array}{lllll}\begin{array}{l}\text { Total } \\ \text { referrals }\end{array} & \text { Category } 1 & \text { Category } 2 & \text { Category } 3 & \begin{array}{l}\text { Unable to } \\ \text { contact }\end{array}\end{array}$

\begin{tabular}{|c|c|c|c|c|c|}
\hline \multicolumn{6}{|l|}{ Coronary intervention } \\
\hline Diagnostic angiogram & 96 & 4 & 15 & 49 & 28 \\
\hline Left and right heart catheterisation & 20 & 1 & 2 & 15 & 2 \\
\hline ? Proceed & 255 & 35 & 50 & 138 & 32 \\
\hline $\mathrm{PCl}$ & 145 & 28 & 32 & 64 & 21 \\
\hline Rotablation & 2 & 1 & 1 & 0 & 0 \\
\hline СТO & 9 & 1 & 1 & 7 & 0 \\
\hline
\end{tabular}

Structural Intervention

\begin{tabular}{|c|c|c|c|c|c|}
\hline Alcohol septal ablation & 1 & 0 & 0 & 1 & 0 \\
\hline Aortic coarctation & 4 & 0 & 0 & 0 & 4 \\
\hline ASD closure & 18 & 0 & 0 & 0 & 18 \\
\hline Balloon aortic valvuloplasty & 1 & 0 & 0 & 1 & 0 \\
\hline Balloon mitral valvuloplasty & 2 & 0 & 0 & 0 & 2 \\
\hline MitrClip & 3 & 1 & 2 & 0 & 0 \\
\hline Paravalvular leak closure & 3 & 1 & 0 & 0 & 2 \\
\hline PF0 closure & 109 & 0 & 0 & 109 & 0 \\
\hline Transcatheter valve implantation* & 98 & 67 & 18 & 13 & 0 \\
\hline Total $^{\star}$ & 766 & 139 & 121 & 397 & 109 \\
\hline
\end{tabular}

${ }^{*}$ All investigations complete.

ASD, atrial septal defect; CTO, chronic total occlusion; PCI, percutaneous coronary intervention; PFO, patent foramen ovale.

pathway integrated best practice recommendations available at the time of its conception and local experiences of dealing with COVID-19 within the cardiology and infective diseases departments. The pathway was proposed prior to the construction of the NHS England and NHS London 'operating framework for urgent and planned services in hospital settings during COVID-19, 3

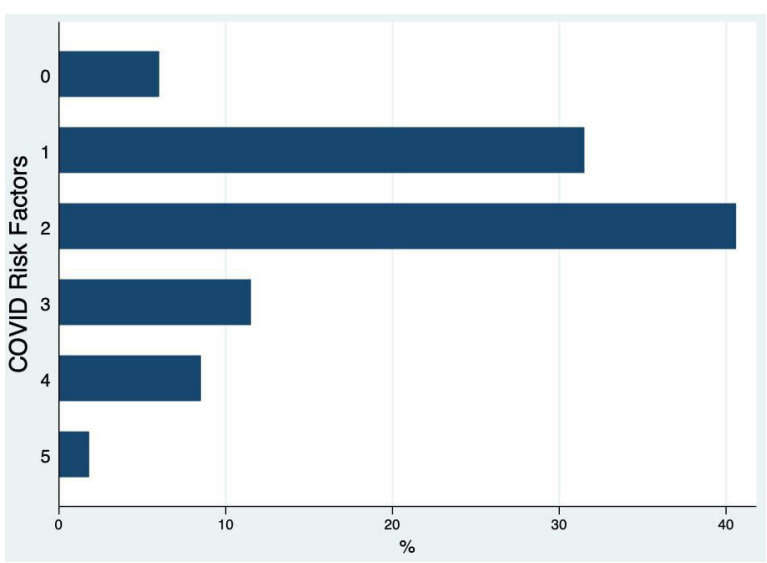

Figure 2 COVID-19 risk factors (combined coronary and structural patients). COVID-19 risk factors: age $>70$ years, Body Mass Index $>35$, renal impairment (estimated glomerular filtration rate<60), cancer, BAME, black, Asian and minority ethnic.

\section{METHODS}

\section{Study population}

All patients on cardiac interventional waiting lists between April and June 2020 at Barts Heart Centre, London, were included in this pilot. Each patient's electronic medical records, referral, and angiographic and adjunctive imaging were reviewed by medical staff. All patients were then contacted to assess risk based on indications (ie, stable angina, severe aortic stenosis and medically managed acute coronary syndrome (ACS)), cardiac symptoms and patient-related adverse features for COVID-19 prognosis (age, renal function, gender, coexistent lung disease and Body Mass Index). Finally, it was discussed whether the patient was agreeable to attending during the pandemic. The patients were placed into three categories, depending on their urgency and risk profile (figure 1):

- Category 1: urgent (to be done as soon as able, ideally within 4-6 weeks).

- Frequent symptoms on minimal exertion.

- Significant worsening symptoms (Canadian Cardiovascular Society (CCS 3-4)).

- Coronary anatomical risk-severe left main stent, proximal left anterior descending (LAD), three vessel disease.

- Coronary bystander disease following ACS.

- Medically managed non-ST segment elevation myocardial infarction (NSTEMI). 


\begin{tabular}{|c|c|}
\hline & $n=101$ \\
\hline Age (year), mean $\pm S D$ & $62.29 \pm 10.12$ \\
\hline Male sex, n (\%) & $84(83.2)$ \\
\hline Black, Asian, minority ethnic, n (\%) & $58(57.4)$ \\
\hline Median BMI (IQR) & $27.55(25.14-29.39)$ \\
\hline \multicolumn{2}{|l|}{ Medical history, n (\%) } \\
\hline Hypertension & $55(54.4)$ \\
\hline Hypercholesterolaemia & $62(61.4)$ \\
\hline Diabetes mellitus & $31(30.7)$ \\
\hline Smoking history & $20(19.8)$ \\
\hline Previous Ml & $31(30.7)$ \\
\hline Previous PCl & $41(40.6)$ \\
\hline Previous CABG & $9(8.9)$ \\
\hline LV ejection fraction (\%), mean \pm SD & $53.70 \pm 7.27$ \\
\hline Serum creatinine $(\mu \mathrm{mol} / \mathrm{L})$, mean $\pm \mathrm{SD}$ & $83.86 \pm 16.33$ \\
\hline Elective procedure, $n(\%)$ & $72(71.3)$ \\
\hline Staged procedure following ACS, n (\%) & $18(17.8)$ \\
\hline Angiogram $\pm \mathrm{PCl}$ for medically managed ACS & $11(10.9)$ \\
\hline Femoral access, n (\%) & $15(14.9)$ \\
\hline \multicolumn{2}{|l|}{ Procedural type, n (\%) } \\
\hline Diagnostic angiogram, $\mathrm{n}(\%)$ & $33(32.7)$ \\
\hline Medical management-no planned interventions & $12(36.4)$ \\
\hline Workup for valve surgery & $1(3)$ \\
\hline Investigations of troponin-positive events & $3(9.1)$ \\
\hline Other surgery & $3(9.1)$ \\
\hline Planned for staged PCI & $3(9.1)$ \\
\hline Referral for CABG/MDT & $11(33.3)$ \\
\hline Diagnostic device used, no therapeutic intervention n (\%) & $14(13.9)$ \\
\hline $\mathrm{PCl}, \mathrm{n}(\%)$ & $54(54.5)$ \\
\hline left anterior descending artery & $31(57.4)$ \\
\hline Left circumflex & $23(42.6)$ \\
\hline Right coronary artery & $12(22.2)$ \\
\hline Grafts & $2(3.7)$ \\
\hline Diagnostic device used to guide $\mathrm{PCl}$ & $31(57.4)$ \\
\hline Functional indices (FFR/iFR) & $5(16.1)$ \\
\hline Intravascular ultrasound & $24(77.4)$ \\
\hline Optical coherence tomography & $2(6.4)$ \\
\hline \multicolumn{2}{|l|}{ Procedural complexity } \\
\hline Multivessel PCl & $11(20.4)$ \\
\hline Number of stents used, mean $\pm S D$ & $1.47 \pm 0.79$ \\
\hline Length of segment treated $(\mathrm{mm})$, mean $\pm \mathrm{SD}$ & $41.63 \pm 20.9$ \\
\hline Bifurcation, n (\%) & $8(14.8)$ \\
\hline Instent restenosis, n (\%) & $4(7.4)$ \\
\hline СT0, n (\%) & $4(7.4)$ \\
\hline Calcium modification, n (\%) & $4(7.4)$ \\
\hline Rotational atherectomy & $3(5.6)$ \\
\hline Intravascular Ilthotripsy & $1(1.9)$ \\
\hline
\end{tabular}

ACS, acute coronary syndrome; BMI, Body Mass Index; CABG, coronary artery bypass; LV, left ventricle; MDT, multidisciplinary team; MI, myocardial infarction; $\mathrm{PCl}$, percutaneous coronary intervention.
- To facilitate non-cardiac emergency treatment (renal/oncology).

- Category 2: symptomatic on exertion but stable symptoms-to be done within 4 months.

- Category 3: stable long-standing symptoms-to be done within 6 months.

\section{Multidisciplinary team (MDT)}

All category 1 patients were reviewed in a virtual MDT meeting (containing interventional consultants and cardiothoracic surgeons if surgical revascularisation options would need to be discussed). This was performed using an online video conferencing system (StarLeaf, Watford, Hertfordshire). The MDT reviewed the patient's risk/ urgency to ensure category 1 status and then weighed up the benefit for the patient to undergo coronary or structural intervention against the potential risk of a day case admission during a pandemic. Patients were then listed in consensus was agreed on the above.

\section{Preassessment}

For infection control purposes, all preassessment appointments were held over the telephone. Patients were further screened for COVID-19 symptoms and exposure at that stage and underwent a standard preassessment consultation. Patients were informed they would have to self-isolate at home for 2 weeks prior to admission. A final preadmission check was performed by an advanced clinical practitioner, 24 hours prior to admission to assess for COVID-19 symptoms (cough, fever and new loss of sense of smell (anosmia)), confirming isolation and checking for new potential COVID-19 exposure. At this stage, a further discussion about the risks and benefits of attending during the pandemic was held with the patient to ensure their understanding of potential additional risk of contracting COVID-19 while in the hospital. This process was standardised for all patients, covering the risk of contracting COVID-19 during the admission and the means to reduce this risk. If patients agreed, this was documented in their electronic medical records, with them given a copy on the day of admission. If all the aforementioned were satisfied, patients were admitted the following day, with second consent performed for the procedure via standard trust consent forms.

\section{Admission}

All patients were met on admission, given a face mask and escorted to a side room on a clean (COVID-19-free) ward. No relatives were allowed to accompany the patient. On arrival on the ward, patients were swabbed for COVID19; staff contact was minimised; and all patient-to-staff contact was made using standard local personal protective equipment guidelines. Patients undergoing percutaneous coronary intervention (PCI) procedures were loaded with $600 \mathrm{mg}$ clopidogrel and $300 \mathrm{mg}$ aspirin on the morning, depending on pre-existing medication. Blood tests were performed on the day of the procedure 
Table 3 COVID-19 screening and admission characteristics for all patients ( $n=143 ; 101$ coronary, 42 structural)

\begin{tabular}{|c|c|c|}
\hline & $\begin{array}{l}\text { Coronary } \\
\text { intervention } \\
(n=101)\end{array}$ & $\begin{array}{l}\text { Structural } \\
\text { intervention } \\
(\mathrm{n}=42)\end{array}$ \\
\hline \multicolumn{3}{|l|}{ Preadmission } \\
\hline MDT discussion & $101(100)$ & $42(100)$ \\
\hline Self-Isolation & $98(97.0)$ & $41(97.6)$ \\
\hline Key worker & $2(2.1)$ & $0(0)$ \\
\hline \multicolumn{3}{|l|}{ Admission } \\
\hline Isolated on ward & $81(80.2)$ & $42(100)$ \\
\hline Stay over 24 hours & $2(2.1)$ & $2(2)$ \\
\hline Overnight stay & $1(1)$ & $32(78)$ \\
\hline 2 days-1 week & $0(0)$ & $5(12.2)$ \\
\hline Greater than 1 week & $1(1)$ & $4(9.8)$ \\
\hline $\begin{array}{l}\text { Brought back for physical review } \\
\text { within } 30 \text { days }\end{array}$ & $3(3)$ & $0(0)$ \\
\hline \multicolumn{3}{|l|}{ Week 2 health questionnaire (EQ5D) } \\
\hline Mobility & $1.75(0.99)$ & - \\
\hline Self-care & $1.45(0.85)$ & - \\
\hline Usual activities & $1.73(0.98)$ & - \\
\hline Pain & $1.745(1.03)$ & - \\
\hline Anxiety/depression & $1.54(0.83)$ & - \\
\hline Total score & $73.30 \pm 21.93$ & - \\
\hline $\begin{array}{l}\text { Notable improvement in symptoms } \\
\text { at } 2 \text { weeks }\end{array}$ & - & $14(45.2)$ \\
\hline \multicolumn{3}{|l|}{ COVID-19 } \\
\hline $\begin{array}{l}\text { Swab or antibody+ve prior to } \\
\text { admission }\end{array}$ & $1(1)$ & $0(0)$ \\
\hline \multicolumn{3}{|l|}{ Admission investigations } \\
\hline Swab+ve on admission & $0(0)$ & $0(0)$ \\
\hline Antibody tests on admission & $36(36 \%)$ & $1(3.2)$ \\
\hline Antibody+ve on admission & $3(8.3 \%)$ & $0(0)$ \\
\hline Symptoms postdischarge & $0(0)$ & $1(3.2)$ \\
\hline
\end{tabular}

MDT, multidisciplinary team; +ve, positive.

using point-of-care testing. The patients underwent their procedure in a cardiac catheter lab and were transferred to and from the ward via a designated clean transfer route. They were subsequently discharged as per routine cardiology intervention practice. Patients were contacted 1 and 2 weeks postprocedure to assess for COVID-19 status, clinical events (MACE) and quality of life (QOL).

\section{RESULTS}

\section{Patient population}

A total of 766 elective patients were reviewed up to 30 June 2020. Of these patients, 527 (68.8\%) were referred for coronary procedures and 239 patients were awaiting a structural procedure. The classification breakdown of telephone assessment can be seen as follows (table 1); a total of $109(14.2 \%)$ were uncontactable during these phone consultation. Of the 527 patients who underwent elective coronary procedure, 132 were discussed in virtual MDT, with 1 patient being excluded due to clinical indication for angiography. In the structural group, 80 patients were discussed, with 13 patients excluded as being symptom free at the time of the review.

\section{COVID-19}

All patients were screened for COVID-19 PCR on admission, although two swabs were lost in transit to the lab and were not processed. All of the 143

Table 4 Structural intervention patient demographics

Elective structural $(n=42)$

Age (year), mean $\pm S D$ $80.90 \pm 5.92$

Male sex, $\mathrm{n}(\%)$ $22(52.4)$

Black, Asian, minority ethnic, $n$ (\%) 4 (9.5)

Height (cm), mean \pm SD

$165.51 \pm 9.90$

Weight $(\mathrm{kg})$, mean \pm SD $75.02 \pm 11.00$

Median body surface area $\left(\mathrm{m}^{2}\right)$ (IQR) $1.81(1.71-1.95)$

Median BMI (IQR) 26.7 (25.04-31.16)

CCS (II-III) 2 (4.8)

NYHA (III-IV)

$30(71.4)$

Medical history, $\mathrm{n}(\%)$

$\begin{array}{lc}\text { Hypertension } & 22(52.4) \\ \text { Hypercholesterolaemia } & 7(16.7) \\ \text { Diabetes mellitus } & 9(21.4) \\ \text { IHD (PCI/CABG/MI) } & 28(66.7) \\ \text { Chronic kidney disease } & 8(19.0) \\ \text { Serum creatinine ( } \mu \text { mol/L), mean } \pm S D & 109.74 \pm 63.89 \\ \text { LV ejection fraction } & 53.98 \pm 10.45 \\ \text { AVA }\left(\mathrm{cm}^{2}\right) & 0.68 \pm 0.18 \\ \text { Peak velocity }(\mathrm{ms}) & 4.24 \pm 0.61\end{array}$

Procedural type, n (\%)

$\begin{array}{ll}\text { TAVI } & 39(92.9) \\ \text { Pulmonary artery intervention } & 0(0) \\ \text { MitraClip } & 2(4.8) \\ \text { Paravalvular leak closure device } & 1(2.4) \\ \text { Complications, } n(\%) & 8(18.2) \\ \text { Permanent pacing } & 4(50) \\ \text { Emergency valve surgery } & 1(12.5) \\ \text { Femoral complication } & 2(25) \\ \text { Myocardial infarction } & 1(12.5) \\ \text { Pulmonary oedema } & 1(12.5)\end{array}$

AVA, aortic valve area; BMI, Body Mass Index; CCS, Canadian Cardiovascular Society; LV, left ventricular; MI, myocardial infarction; NYHA, New York Heart Association; PCI, percutaneous coronary intervention; TAVI, transcatheter aortic valve intervention. 
patients with tested samples were PCR swab negative. COVID-19 antibody testing became available on 8 June 2020 with 37 subsequent patients tested on admission. Of these 37 patients, $8.1 \%$ (3 patients) were antibody positive (but were all swab negative on admission). All of the positive patients had COVID-19 symptoms in the last few months following $\mathrm{A}+\mathrm{E}$ attendances at district general hospitals due to chest pain. In terms of COVID-19 risk, over $93 \%$ had one or more moderate risk factors, with the breakdown shown in figure 2.

\section{Elective coronary procedures}

A total of 125 coronary patients were scheduled for admission between 20 April 2020 and 19 June 2020, with 22 patients cancelled preadmission (1 due to exposure to COVID-19, 6 declined admission following discussion regarding COVID-19 risk, 7 due to clinical reasons, 3 presented acutely as ACS and 6 declined the TCI (to come in) date). In total, 101 patients were admitted for coronary procedures (3 having two procedures during the time frame), with the baseline demographic and procedural information for these patients shown in table 2. All patients had been discussed in the virtual MDT prior to admission; three patients did not fully self-isolate prior to admission (two were key workers and one had a previous COVID-19+ swab (3 months earlier) prior to admission and declined to self-isolate).

The indication for the procedure was angina in $71.3 \%$ of the patients and staged procedure following ACS in $17.8 \%$ and $10.9 \%$ of the patients were following an initially medically managed ACS. Fiftyfour patients $(54.5 \%)$ underwent PCI with a total of 33 patients undergoing diagnostic angiography with the remaining 31 cases using diagnostic devices to facilitate coronary intervention. There were only four immediate procedural complications, three haematomas and one coronary dissection. Ninetyeight patients $(94.2 \%)$ were discharged on the same day with $6(5.8 \%)$ having overnight stays due to coronary dissection, ${ }^{4}$ vascular complications,${ }^{5}$ observation post-CTO ${ }^{6}$ and for urgent $\mathrm{CABG}^{4}$ (table 3 ).

\section{Elective structural procedures}

A total of 42 patients underwent urgent elective structural procedures, with 39 patients undergoing transcatheter aortic valve intervention, 2 undergoing MitraClip and 1 undergoing paravalvular leak closure. The baseline demographics and procedural information for these patients can be seen in table 4 and admission in table 3. Eight patients had complications during structural procedure, with four patients requiring urgent pacing, one requiring urgent valve surgery and two with femoral access complications. The majority of patients had one night admission with nine elective patients admission being greater than 3 days.

\section{Follow-up}

\section{Coronary}

One-week telephone follow-up was performed for the first 39 patients but was stopped after this due to time restraints and increased elective admissions. No patients had COVID-19 symptoms at that point. At 2 weeks of follow-up, all patients had no symptoms of COVID-19, with no confirmed COVID-19 cases. QOL was recorded for 97 patients, with a mean EQ5D-VAS of 73.5 \pm 19.8 (range 30-100). No MACE events were recorded during the 2-week follow-up period $(0 \%)$.

\section{Structural}

At 2 week of follow-up, one patient reported a fever but was COVID-19 swab negative and was treated for a community acquired chest infection by their GP. One patient was admitted to the hospital for non-cardiac issues and three patients reported worsening lethargy postprocedure; however, 14 patients $(45 \%)$ noticed immediate improvement in symptom benefit.

\section{DISCUSSION}

The reintroduction of a safe elective pathway to facilitate the treatment of cardiovascular disease is paramount in the current COVID-19 pandemic. ${ }^{6}$ In this pilot study, we have demonstrated that patients can be brought in safely for urgent elective interventional procedures to a centre where COVID-19-positive patients are also being managed. Measures such as effective case triaging, thorough history taking and patient screening, periods of self-isolation and careful well-planned pathways within the hospital, including designated COVID-19-free areas, meant that all patients were treated without contracting COVID-19 infection. The hiatus of elective waiting lists can lead to increased stress and anxiety for patients or can result in acute care hospital admissions, meaning increased risk of COVID-19 exposure. ${ }^{5}$ Importantly, this pathway provides an effective solution to this clinical need in the current climate for patients who are happy to attend after being adequately informed and formally consenting to COVID-19 risk.

A key aspect of the pathway was to minimise patient hospital time and visits, that is, telephonic preadmission, point-of-care blood testing, on-the-day swabs/ drug loading and minimising length of stay, all to reduce possible COVID-19 exposure. The coronary interventional patients had on average less than 12 hours' stay within the hospital, with the structural patients staying just over 24 hours within the hospital with the majority discharged on day 2. However, despite these protocols developed to minimise the risk of COVID-19 transmission, the risk cannot be completely eliminated. It is imperative for additional risk of contracting COVID-19 to be highlighted and for this to be formally documented in the consenting process. 
One issue with this pathway is the timing and use of nasal swab for preprocedural testing rather than antibody testing. Establishing COVID-19 status by nasopharyngeal swab was essential to manage patients staying overnight in appropriate areas within the hospital. There were only small numbers of coronary patients requiring admission overnight, but this was standard practice for structural cases. However, swab results were not available during admission or often predischarge. Antibody testing was introduced in the interventional group after 64 patients, with only around $8 \%$ of patients having positive results. With the introduction of antibody testing nationwide, there may be a role for these tests (swab and antibody) to be done prior to admission to assess patients' COVID-19 status prior to their procedure. Despite no swabbing being undertaken preprocedure, not a single patient was COVID-19 positive on admission, highlighting the robustness of our 2-week isolation and screening questionnaires. However, aspects of this pathway, although essential at the time, are difficult to maintain as normal service slowly resumes. Isolation for 2 weeks prior to admission was straightforward with the majority of cardiac patients shielding. However, the psychological effects of lockdown especially in the elderly population has been well documented, ${ }^{7}$ and as this is relaxed on a population level, it is likely that the pathway will need to evolve, for example, 7 days of self-isolation with routine PCR swapping preprocedure (1-3 days).

It is natural that both cardiologists and patients are apprehensive about restarting elective services, with one of the biggest challenges among patients awaiting elective procedures being the fear of contracting COVID-19. Some studies have reported up to $50 \%$ of patients awaiting orthopaedic procedures, wanting to wait until postpandemic, although no data exists for elective cardiac procedures. ${ }^{4}$ In our pathway, the use of cardiologists and advanced nurse practitioners to assess and contact patients resulted in correct patient identification and reassurance to these patients rather than clerical staff. In addition, the allocation of a single dedicated allied nurse professional to lead the admission and follow-up improved patient care and reassurance. In many cases, issues were identified in follow-up that helped expedite or facilitate patient care postprocedure, all of which were an inevitable consequence of reduced services during a pandemic, that is, availability of cardiac rehabilitation.

It is known that patients with pre-existing cardiovascular disease are likely to have comorbidities which are associated with poorer clinical outcomes in confirmed SARS-CoV-2 cases. ${ }^{89}$ Additionally the majority $(>90 \%)$ of patients treated had one or more moderate adverse features, in addition to cardiac disease such as age over $>70$ years or renal dysfunction, a truly reflective population of those requiring treatment. Despite this risk, no patient reported
COVID-19-like symptoms postadmission at the 2-week follow-up with no confirmed cases and no adverse cardiac events. Importantly, at this point, a significant increase in QOL was also seen despite the ongoing pandemic, highlighting the importance of reintroducing these services. As we have demonstrated this safe pathway for cardiovascular patients, this can be used as a blueprint for the reintroduction of other importance elective treatments, that is, orthopaedic surgery.

\section{CONCLUSION}

Restarting elective cardiac services during the COVID-19 pandemic is essential, given the burden of disease and its potential impact. This pilot demonstrates that with careful planning and structured protocols, interventional cardiac procedures can be safely delivered with no patient developing COVID-19 infection, despite high-risk features in the patient cohort. Despite the risks being minimised, they cannot be completely eliminated, and it is imperative patients are counselled and consented formally prior to their procedures.

\section{Twitter Michael Mullen @bartstrctural}

Acknowledgements We acknowledge the cardiology advanced clinical practitioners (Oliver Casey-Gillman and Lizzie Moore), the transcatheter aortic valve intervention specialist nurses (Helen Queenan, Melanie Jerrum and Kerry Bedford) and the Cath Lab management team (Rob Hall and Simon Cook) for their contribution to the pilot. Finally, we thank all the staff of Barts Heart Centre for their hard work and professionalism during the COVID-19 outbreak, and for the NHS patients who have been very supportive throughout this difficult time.

Contributors Planning: SH, GM, GF, JL, AM and DJ. Conduct: SH, KC, FC, KR, MA, AW, MO, OG, MM, AB, ES, AM and DJ. Overall content: SH, AM and DJ.

Funding The authors have not declared a specific grant for this research from any funding agency in the public, commercial or not-for-profit sectors.

Competing interests None declared.

Patient consent for publication Not required.

Ethics approval Local discussion within the Barts Health NHS Trust was performed prior to the initiation of the programme.

Provenance and peer review Not commissioned; externally peer reviewed. Data availability statement No data are available.

Open access This is an open access article distributed in accordance with the Creative Commons Attribution Non Commercial (CC BY-NC 4.0) license, which permits others to distribute, remix, adapt, build upon this work non-commercially, and license their derivative works on different terms, provided the original work is properly cited, appropriate credit is given, any changes made indicated, and the use is non-commercial. See: http://creativecommons.org/licenses/by-nc/4.0/.

\section{ORCID iDs}

Stephen Hamshere http://orcid.org/0000-0002-8823-1019

Michael Mullen http://orcid.org/0000-0002-4911-3511

\section{REFERENCES}

1 Dashboard. WHOCOVID.

2 Wu Z, McGoogan JM. Characteristics of and Important Lessons From the Coronavirus Disease 2019 (COVID-19) Outbreak in China: Summary of a Report of 72314 Cases From the Chinese Center for Disease Control and Prevention. JAMA 2020;323:1239-1242.

3 England NHS. Operating framework for urgent and planned services in hospital settings during COVID-19. 
4 Chang J, Wignadasan W, Kontoghiorghe C, et al. Restarting elective orthopaedic services during the COVID-19 pandemic. Bone Jt Open 2020;1:267-71.

5 da Fonseca VBP, De Lorenzo A, Tura BR, et al. Mortality and morbidity of patients on the waiting list for coronary artery bypass graft surgery. Interact Cardiovasc Thorac Surg 2018;26:34-40.

6 Clerkin KJ, Fried JA, Raikhelkar J, et al. COVID-19 and cardiovascular disease. Circulation 2020;141:1648-55.
7 Plagg B, Engl A, Piccoliori G, et al. Prolonged social isolation of the elderly during COVID-19: between benefit and damage. Arch Gerontol Geriatr 2020;89:104086.

8 Khan IH, Zahra SA, Zaim S, et al. At the heart of COVID-19. J Card Surg 2020;35:1287-94.

9 W-j G, W-h L, Zhao Y. Comorbidity and its impact on 1590 patients with COVID-19 in China: a nationwide analysis. European Respiratory Journal Eur Respir J 2020;55:2000547. 